\title{
Reinforcement of rat hippocampal LTP by holeboard training
}

\author{
Shukhrat Uzakov, Julietta U. Frey, and Volker Korz ${ }^{1}$ \\ Department of Neurophysiology, Leibniz Institute for Neurobiology, D-39118 Magdeburg, Germany
}

\begin{abstract}
Hippocampal long-term potentiation (LTP) can be dissociated in early-LTP lasting 4-5 $\mathrm{h}$ and late-LTP with a duration of more than $8 \mathrm{~h}$, the latter of which requires protein synthesis and heterosynaptic activity during its induction. Previous studies in vivo have shown that early-LTP in the dentate gyrus can protein synthesis-dependently be transformed (reinforced) into late-LTP by the association of arousing novel environmental stimuli. Here we show that consolidation of spatial memory also reinforces early-LTP in the dentate gyrus. Both memory consolidation and LTP-reinforcement depend on protein synthesis. Four groups of animals were trained by five, seven, eight or 10 trials, respectively, to recognize a fixed pattern of baited holes. The last trial was performed $15 \mathrm{~min}$ after tetanus. Errors of long-term reference memory during the last trial were significantly decreased only in the eight- and 10-trial experimental groups compared to pseudo-trained animals. In correlation to this learning effect we found a reinforcement of early-LTP only in these experimental groups compared to controls. The data suggest that the synthesis of new proteins required for spatial reference-memory formation also contributes to LTP maintenance in the hippocampal dentate gyrus.
\end{abstract}

Long-term potentiation (LTP), a widely studied cellular model for learning and memory formation, can-similar to memory forma tion (Flexner et al. 1965; Grecksch and Matthies 1980; Izquierdo et al. 2002) — be dissociated into an early phase and a late phase. We use the terms "early-LTP" for an initial phase of LTP with a 4-5-h duration that is protein synthesis-independent, and "late-LTP" for a late phase that protein synthesis-dependently maintains over more than $8 \mathrm{~h}$. As pointed out by Kelleher III et al. (2004), these temporal phases of LTP were initially described by Krug et al. (1984) and Frey et al. (1988) and were confirmed by others in vivo (Otani and Abraham 1989; Otani and Ben-Ari 1993) and in vitro (Huang et al. 1994). It was shown that this kind of early-LTP is reinforceable in vivo; that is, it can protein synthesisdependently be transformed into late-LTP (Matthies et al. 1986), by a temporal-related second heterosynaptic input (Seidenbecher et al. 1997; Frey et al. 2001; Straube et al. 2003b). The induction of this form of LTP, in contrast to a short-term potentiation (Frey and Morris 1997), sets a tag at the potentiated synapses that then can profit from plasticity-related proteins, the synthesis of which was induced by the modulatory heterosynaptic inputs. Thus, the processing of the newly synthesized plasticity-related proteins by the tagged synapses allows the transformation of early-LTP into late-LTP. This is essential for reinforcement processes. There is a large body of literature reporting that the maintenance of memories also requires heterosynaptic activation (for review, see Bailey et al. 2000). Late-LTP is considered to be related to long-term memory, whereas early-LTP may represent short-term memory traces (Bach et al. 1999; Jones et al. 2001). Therefore we aimed at correlating consolidation processes on the behavioral level and the cellular level by learning-related induction of the abovedefined LTP. Although other forms of early-LTP with a faster decay than that used in this study have been described (Impey et al. 1996; Abel et al. 1997), reinforcement of these forms of in vivo

\footnotetext{
'Corresponding author.

E-mail korz@ifn.madgeburg.de; fax 49-391-62-63-421.

Article published online ahead of print. Article and publication date are at
} http://www.learnmem.org/cgi/doi/10.1101/lm.89305.
early-LTP has not been shown. Thus, we refer here to the terms of early- and late-LTP as described above.

Modulatory heterosynaptic inputs to the dentate gyrus (DG) in vivo can be provided by stimulation of brain regions involved in the processing of emotionally relevant information such as the basolateral amygdala (Frey et al. 2001; Almaguer-Melian et al. 2003) or the medial septum (Frey et al. 2003) and by behaviorally relevant arousing new information of novel environments (Korz and Frey 2003a; Straube et al. 2003a,b). Reinforcement by novelty detection typically is a rapid process that occurs after a single exposure to the arousing situation, and can be blocked by a brief pre-exposure to the stimuli making them familiar (Straube et al. 2003a). The term "emotional tagging" was introduced to characterize this form of LTP-reinforcement related to the rapid consolidation of relevant new information (Richter-Levin and Akirav 2003). It was suggested that the main modulatory input for emotional reinforcement is of noradrenergic nature (Seidenbecher et al. 1997; Straube et al. 2003b). A protein synthesis-dependent role of glucocorticoids in reinforcement of DG-LTP by activation of the corticosterone-binding mineralocorticoid receptor (that acts as a transcription factor) after a brief stressful event was also found (Korz and Frey 2003a).

In this study, we addressed whether learning of complex spatial tasks can also reinforce hippocampal early-LTP which then could be considered as "cognitive reinforcement," in contrast to "emotional reinforcement." The strength of the heterosynaptic input triggered by holeboard learning can be regulated by the intensity of training or the complexity of the task in order to induce acquisition-related processes or consolidation of spatial memories. The absence or the occurrence of reinforcement during different stages of learning should then indicate the synthesis of proteins being related to both specific memories and the maintenance of LTP, thus providing evidence for consolidation on the behavioral and the cellular level. We chose a holeboard paradigm for spatial learning in which the transient working memory and the lasting reference memory can be evaluated in parallel and be related to LTP. Additionally, by using a holeboard with a sufficient amount of holes, the complexity of the task can be effec- 
tively fine-tuned by using a wide range of simple to very complex patterns of baited holes. In future studies, this model can be used for the identification of proteins critically involved in learning and plasticity.

\section{Results}

The regular distribution of holes within the board along with unspecific explorative activity of the rats causes random hole visits and inspections that are not related to spatial learning. To discriminate between random reference and working errors made during exploration of the board from those made during the task acquisition, a group of animals were pseudo-trained during 10 trials; i.e., the pattern of baited holes was changed after each trial. Thus, the pseudo-trained group served as a control for random errors, and all experimental groups were compared to this group. The experimental 10-trial group showed significantly decreased latencies compared to pseudo-trained rats from Trial 8 on (Fig. $1 \mathrm{~A}, P<0.01$, each) and fewer reference-memory errors during Trial 10 (Fig. 1B, $P<0.001$ ), but did not differ in workingmemory errors $(P>0.05$, each). The comparison of the last trial between experimental and control groups (Fig. 2) revealed a significant difference in latencies $\left(F_{(5,48)}=56.59, P<0.001\right)$ and in reference-memory errors $\left(F_{(5,48)}=9.02, P<0.001\right)$ but not in working-memory errors $\left(F_{(5,48)}=1.93, P>0.1\right)$. Post hoc tests against pseudo-trained animals revealed reduced referencememory errors only for the eight- $(P=0.001)$ and 10-trial $(P<0.001)$ experimental groups, and for the seven-, eight- and 10 -trial experimental groups significantly decreased latencies $(P<0.001$, each). Between the two latter experimental groups we found no difference in either of the measured behavioral parameters $(P>0.1$, each).

Early LTP was induced $15 \mathrm{~min}$ before starting the last holeboard trial for the different groups, in order to test whether the different stages of task acquisition and memory formation indicated in the behavioral parameters have different impacts on the maintenance of LTP. We found an overall difference in LTP between groups $\left(F_{(9,80)}=11.73, P<0.001\right)$, with enhanced population spike amplitudes at $24 \mathrm{~h}$ only for the eight- $(P<0.001)$ and 10-trial $(P=0.001)$ experimental animals compared to chamber controls (Fig. 3A). Thus, the analysis of LTP time courses revealed a reinforcement of early-LTP only in those groups that showed significantly reduced reference-memory errors compared to
A

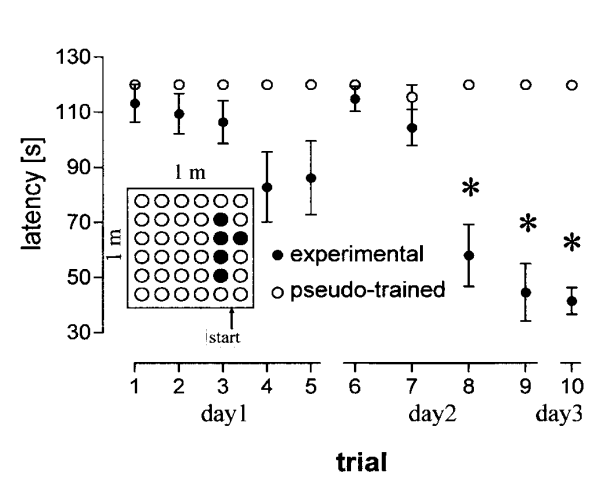

B

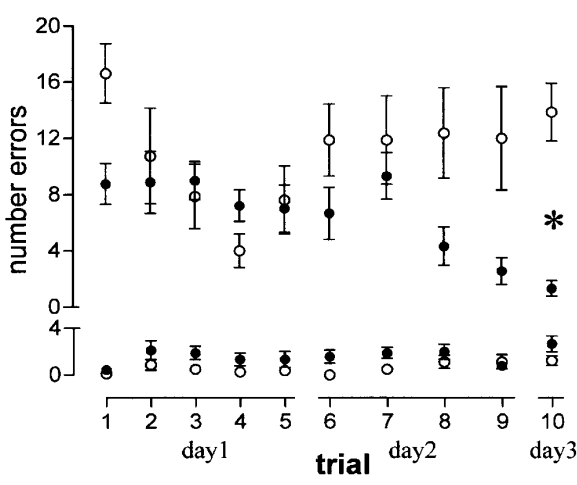

Figure 1. The second training session is crucial for food pellet pattern recognition. Ten-trial experimental animals show decreased latencies (the time needed to find all pellets in seconds) compared to pseudo-trained animals from the eighth trial on $(A)$; the inset indicates the holeboard with the pattern of baited holes $(\bullet)$ and the animals' start position used during training. Experimental animals made significantly fewer reference-memory errors ( $B$, upper) during Trial 10 compared to pseudo-trained animals. Regarding working-memory errors, no difference between the two groups could be noted ( $B$ lower). Abscissa indicates the amount of trials performed on each experimental day. Data are means \pm s.e.m. *: Significant differences after Bonferroni correction. pseudo-trained animals. In contrast, significantly reduced latencies alone with still increased reference errors as shown by the seven-trial experimental group, do not correlate with maintained LTP. Interestingly, the pseudo-trained animals that could not form a reference memory, in terms of pattern recognition, showed not only no reinforcement but a depotentiation (Fig. 3B) as indicated by significantly reduced population-spike amplitude (PSA) compared to chamber controls $(P=0.012)$. Because the animals were food-deprived shortly before and during the experiments, food consumption in the holeboard could have a rewarding effect. Water consumption after water deprivation has been found to reinforce DG-LTP (Seidenbecher et al. 1997). Therefore, to test whether a possible food reward may interfere with learning and memory effects on hippocampal LTP, we employed the food control group. In these animals we noted no reinforcement but a slight, not significant $(P>0.05)$ decrease of PSA $2 \mathrm{~h}$ after holeboard exposure (Fig. 3B). Another possible interfering variable is the detection of novelty that has been shown to reinforce DG-LTP (Korz and Frey 2003b; Straube et al. 2003a). Such an effect was not found in the present study, as indicated by the novelty group showing no difference compared to chamber controls $(P>0.1)$. Due to the different training protocols, experimental animals that received higher numbers of trials possibly are better habituated to the experimental procedure at the timepoint of tetanization compared to the animals that experienced only a few trials. This difference could result in different stress responses to the transference to the holeboard, and that in turn could have different modulatory effects on LTP-maintenance. To test for this, we measured the serum corticosterone levels of animals that were holeboard naive (novelty group) or that received 10 training trials, respectively, compared to food deprived and non-deprived untreated animals (Fig. 4). We found significant differences between groups $\left(F_{(3,26)}=17.89, P<0.001\right)$ with increased titers of both experimental groups compared to controls (novelty group, $P=0.007$; 10 -trial group, $P=0.004$ ) but no difference between the experimental groups $(P>0.8)$. Food deprivation alone elevates the serum corticosterone levels, as indicated by the increased titers of the deprived animals compared to the nondeprived rats $(P=0.002)$.

To test the hypothesis formulated above, that is, that the occurrence of reinforcement during the formation of specific memories indicates the synthesis of new proteins related to both memory formation and the maintenance of LTP, we blocked protein synthesis during training. In a 10trial experiment, anisomycin, a reversible translation inhibitor, was applied before the second training session, which is critical for pattern recognition as indicated by the learning curve of the former experiments (Figs. 1, 2). In addition, applying the drug $24 \mathrm{~h}$ before tetanization should avoid drug-specific effects which may mask learning-specific modulations on the induction and maintenance of LTP. As indicated in Figure 5 , we found no reinforcement of early-LTP in drug-treated rats compared to rats treated with saline $(P<0.001)$. Animals that received anisomycin needed longer times to find all pellets from the eighth trial on compared to controls $(P<0.001$ each; Fig. 5B) or did not find all pellets. Additionally, anisomycin-treated animals made more reference-memory errors during the tenth trial $(P<0.001 ;$ Fig. 5C) compared to 


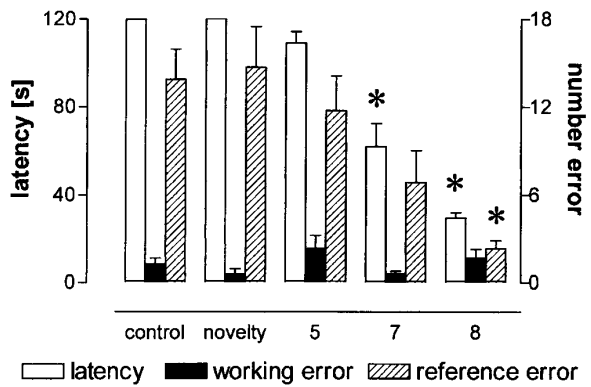

Figure 2. A sudden improvement in reference memory was observed between training Trials 7 and 8 . The seven- and eight-trial experimental groups exhibited lower latencies, but only the eight-trial group made fewer reference-memory errors than pseudo-trained animals during the last training trial. The left ordinate shows the latency to find all pellets; the right ordinate indicates the number of errors. Data are means \pm s.e.m. *: Significant differences.

saline controls. In no case was a difference in working-memory errors between experimental and control groups noted $(P>0.1$, each).

\section{Discussion}

We found a protein synthesis-dependent reinforcement of early LTP only in animals that also exhibited significant improvements in the number of reference-memory errors but not in working-memory errors (cf. Jaffard et al. 1996; Kikusui et al. 2000). The dissociation between working- and reference-memory error performance can be explained by the simple spatial pattern with only a few baited holes in a line. This lowers the probability of making working-memory errors. The last parameter indicates differences in short-term memory, since the reference situation for this kind of error changes from trial to trial. In contrast, reference-memory errors reflect long-lasting spatial memory, because the reference frame remains stable over trials. This suggests that late-DG-LTP and cellular/molecular mechanisms involved in memory formation may share some common features (cf. Jones et al. 2001). mitogen-activated protein kinase ERK in the DG of rats during both sustained LTP and spatial training in a water maze. Induction of late-LTP in the DG evokes a biphasic response in CREB phosphorylation in rats (Schulz et al. 1999) that is very similar to that evoked after high stress induced by forced swimming (Bilang-Bleuel et al. 2002).

Our control groups addressed confounding factors that are known to be involved in the reinforcement of early-DG-LTP. A brain temperature effect on field potentials due to increased activity, as reported by Moser et al. (1994) can be ruled out, because the pseudo-trained animals showed no difference in their overall activity compared to experimental animals, as indicated by the total number of hole visits. A reinforcing effect of novelty detection (Kitchigina et al. 1997) and actual or anticipated food reward that has been found in water-deprived rats after water supply (Seidenbecher et al. 1997) can be ruled out by our novelty and food control groups, showing no difference compared to the chamber control group. This is in seeming contrast to our earlier studies (Straube et al. 2003a,b) reporting that novelty-exposure reinforces early-LTP. However, reinforcement was strongly time-dependent, so that we found no effect on early-LTP when a novel environment was explored 15 min after tetanus (the time window we used during the present study). Besides this there are several other fundamental differences between the former and the present studies: First, in the earlier studies the animals were allowed to explore a novel environment voluntarily, whereas in this study the animals were transferred to the holeboard by hand. Brief handling has been shown to depotentiate an induced early-LTP (Korz and Frey 2003a), so that at best a reversal of depotentiation was induced by the holeboard experience. Second, in contrast to the former studies, animals in the present study were food-deprived, which results in chronically elevated circulating corticosterone titers (cf. Kiss et al. 1994; Heiderstadt et al. 2000; Jurcovicova et al. 2001). The last fact at the same time makes the present study not comparable to another study (Korz and Frey 2003a) in which we reported a reinforcement of LTP by brief acute swim stress 15 min after tetanus. This reinforcement was dependent on the activation of mineralocorticoid receptors (MRs) by binding of corticosterone. Chronically elevated corticosterone levels, however, have a

\section{Similarities and differences}

\section{to other studies}

The results from studies that aimed to relate spatial cognition in different learning paradigms to LTP in CA1 or DG of the hippocampus are contradictory (Jeffery 1997). Saucier and Cain (1995) noted normal spatial learning in the water maze in nonspatially pre-trained rats despite blockade of NMDAR-dependent DG-LTP, whereas others provided evidence that spatial learning is correlated with hippocampal LTP (Richter-Levin et al. 1994; Bach et al. 1999; Schulz et al. 2002). In addition, there is evidence that long-term depression (LTD) is related to learning and memory (Braunewell and Manahan-Vaughan 2001; Nakao et al. 2002; Kemp and Manahan-Vaughan 2004). On the molecular level there are also some parallel findings during LTP and spatial training. Gooney et al. (2002) found increased phosphorylation of the tyrosine kinase receptor TrkB and
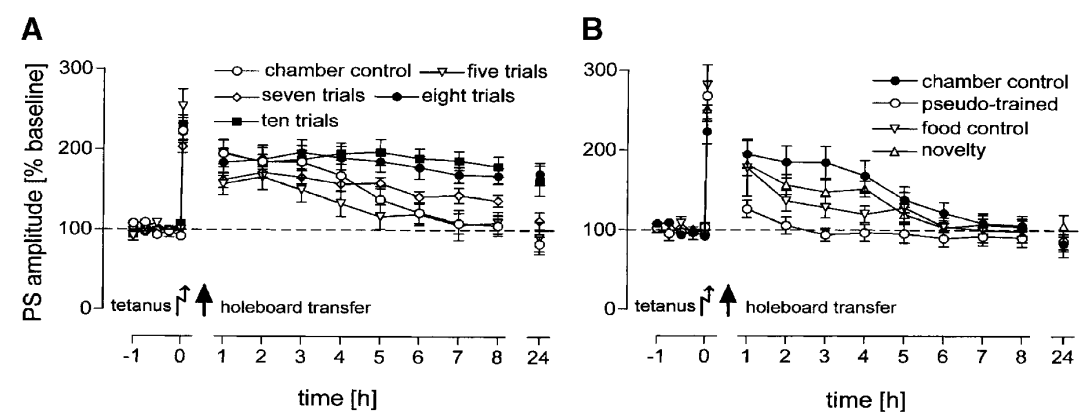

C

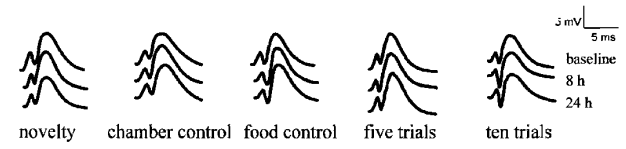

Figure 3. Hippocampal LTP is reinforced by holeboard learning. Early LTP is reinforced after eightand 10-trial training only $(A)$, indicated by significantly increased population spike amplitudes (PSAs) at the 24-h timepoint compared to chamber controls. Food reward and novelty perception had no effect on early-LTP, whereas pseudo-training induced depotentiation (B). Ordinates give the PSAs as percent change from baseline. Representative analog traces of PSAs are given for baseline recordings, the 8-h and 24-h timepoints for individual animals of the different groups (C). Horizontal broken lines indicate baseline. Data are means \pm s.e.m. 


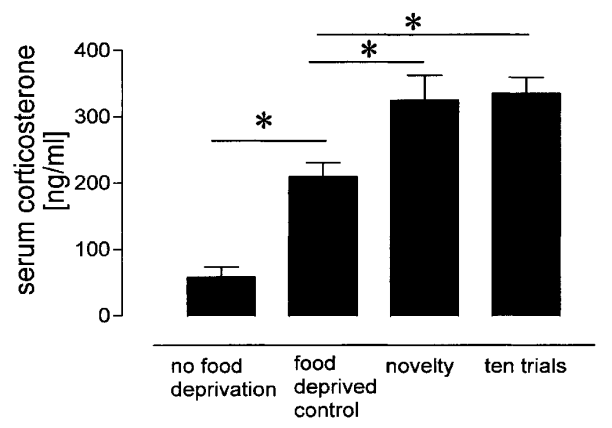

Figure 4. Food deprivation causes a chronic increase in serum corticosterone. Food-deprived unmanipulated rats show chronically increased corticosterone levels compared to nondeprived rats. Transfer to the holeboard causes a slight but significant increase in corticosterone irrespective of the number of trials received (novelty vs. 10 trials). Data are means \pm s.e.m. *: Significant differences.

strong impact on the regulation of MRs as well as glucocorticoid receptor densities and other cellular processes (Duffy et al. 2001; Meyer et al. 2001; Sebaai et al. 2001; Karandrea et al. 2002; Hugin-Flores et al. 2004; Shors 2004). Thus, even the chamber control animals in this study are different in their stress and cellular status compared to control animals used in the previous studies. In addition, a brief pre-exposure to the stressful stimuli in the swim stress experiments blocked the reinforcement of early-LTP despite similar corticosterone levels (Korz and Frey 2003b). Nevertheless, a modulation of DG-LTP by stress in the present study is unlikely, because we found no differences in serum corticosterone levels between experimental groups, but, due to the increase in corticosterone titers in response to the holeboard transfer, an effect cannot completely be ruled out.

\section{Reference memory and LTP reinforcement}

As pointed out, long-term memory requires the synthesis of new macromolecules. It has been shown that memory consolidation depends on heterosynaptic activation that induces protein synthesis: noradrenergic (Przybyslawski et al. 1999; Tronel et al. 2004), dopaminergic, serotonergic, and cholinergic (Castellano et al. 1999; Barros et al. 2001; Phillips et al. 2004). This corresponds to the view that associative processes are required for the maintenance of hippocampal LTP. Different heterosynaptic inputs, such as noradrenergic (Frey et al. 2001, 2003; Straube et al. 2003b), dopaminergic (Manahan-Vaughan and Kulla 2003), or serotonergic (Kulla and Manahan-Vaughan 2002) have been found to modulate hippocampal DG-LTP and depotentiation. In the present study, a heterosynaptic input may be activated by the rapid consolidation of spatial memory during or after Trial 8 , inducing the synthesis of plasticity-related proteins, which then leads to a transformation of the time-related electrically induced early-LTP into a late-LTP in another synaptic input. Future studies will be conducted to test this hypothesis by applying specific receptor inhibitors during training. Thus, the modulatory input involved in cognitive LTP reinforcement remains to be identified, but it has been pointed out that different inputs are probably related to different types of information processing and memory formation (Murchison et al. 2004; for an overview see Myhrer 2003). Although dependent on protein synthesis, decreased latencies alone with still elevated levels of referencememory errors are insufficient to reinforce early-LTP, in contrast to the establishment of correct spatial relations indicated by decreased reference-memory errors. This suggests a specific heterosynaptic activation related to the formation of reference memory and to LTP reinforcement.

Here, the pseudo-trained animals were unable to establish and consolidate a reference memory throughout the training, in contrast to the animals trained on a fixed pattern of pellets. Consequently, the pseudo-trained group showed no reinforcement of hippocampal LTP. Surprisingly, we noted not only no reinforcement but a depotentiation, a reversal of LTP that is comparable to that which we observed in animals that were only handled and then transferred back to the recording chamber (Korz and Frey 2003a). Processes of depotentiation and LTP decay (Doyère et al. 1997; Villarreal et al. 2001; Manahan-Vaughan and Kulla 2003; Woo and Nguyen 2003) as well as that of erasing spatial memories (Villarreal et al. 2001) are regarded as active processes rather than a passive run-down of maintaining mechanisms. Therefore, in our study depotentiation may reflect an adjustment to avoid the storage of behaviorally irrelevant information within hippocampal neuronal circuits (Rosenzweig et al. 2002). Contextdependent modulation of LTP, reinforcement or depotentiation, may serve as a general active mechanism for the specific selection of memories to be consolidated (Doyère et al. 1995; Morris and Frey 1997; Kentros et al. 2004).
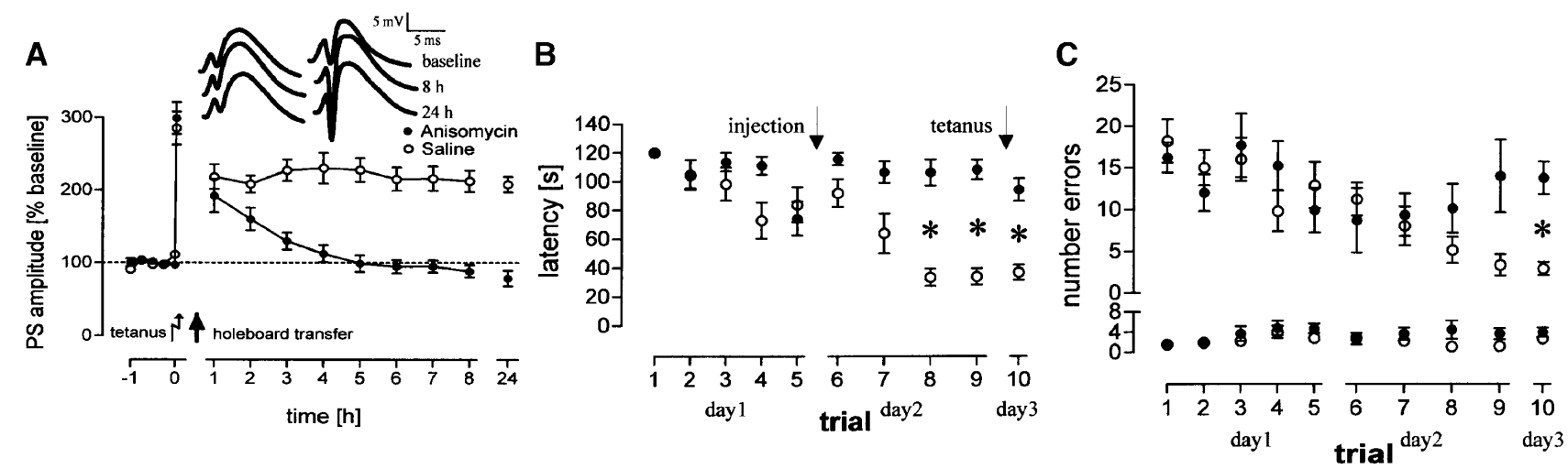

Figure 5. Reinforcement of LTP, decreased latencies, and the formation of a reference memory are protein synthesis-dependent. Anisomycin blocks the reinforcement of LTP $(A)$; the insets show representative analog traces of PSAs at indicated timepoints for an anisomycin-treated (left) and a saline-treated (right) animal. Ordinate gives the PSAs as percent change from baseline. The aquisition of the task is unaffected as indicated by longer latencies in anisomycin-treated rats compared to controls only during the eighth to tenth trials $(B)$. The consolidation of reference memory was affected in drug-treated rats, as indicated by increased reference-memory errors ( $C$, upper) during the tenth trial compared to controls. The groups showed no differences in working-memory errors $(C$, lower). Abscissas in $B$ and $C$ give the number of trials during each experimental day. Arrows indicate the time of drug injection or tetanization, respectively. *: Significant differences after Bonferroni correction. 


\section{Consolidation or reconsolidation of spatial memory?}

Reconsolidation, a new consolidation of memories during their retrieval (Sara 2000) that has been shown to be protein synthesisdependent (Litvin and Anokhin 1999; Nader et al. 2000; Debiec et al. 2002), may also have played a role in the present study, in two ways: (1) reconsolidation of long-term memories formed during the first day of training could be prevented by the application of anisomycin before the second training; and (2) reconsolidation of long-term memories formed during the second training session rather than consolidation of newly formed memories during the last training reinforces hippocampal LTP. That hippocampus-independent memories again become hippocampus-dependent by their reactivation has been shown by Debiec et al. (2002). It cannot be concluded from the present data whether the reinforcement is driven by consolidation, reconsolidation, or both processes.

Our data show that rat hippocampal DG-early-LTP can be reinforced into late-LTP by their learning with a holeboard, and that this effect is protein synthesis-dependent. To verify the specific effect of a spatial component of cognition or other related learning processes, future experiments will be conducted to verify the current findings using different spatial learning paradigms, e.g., the water maze.

\section{Materials and Methods}

All experiments were carried out according to the national animal care guidelines and with permission of the regional council of Saxony-Anhalt. Male Wistar rats (8-wk-old) from the breeding colony of the institute were kept under a $12 \mathrm{~h} / 12 \mathrm{~h}$ light regimen with lights on at 7:00 in standard cages $(40 \mathrm{~cm} \times 25 \mathrm{~cm} \times 18$ $\mathrm{cm})$, the ground covered with commercial bedding material (ssniff, wood spans). The animals were fed with food pellets (ssniff, R/M-H, Soest); tap water was given ad libitum.

\section{Surgery and electrophysiological recording}

Rats were anesthetized with Nembutal (40 mg/kg, i.p.). A monopolar recording electrode (insulated stainless steel, $125 \mu \mathrm{m}$ dia) was implanted stereotaxically into the hilus (coordinates: AP -2.8 , L 1.8 from bregma, 3.2-3.5 ventral from dura) of the dentate gyrus (DG); a bipolar stimulation electrode was implanted into the medial perforant path (AP -6.9, L 4.1, 2.2-2.5 ventral from dura), and in pharmacologically treated rats, a cannula (coordinates AP -0.8 , L 1.6 from bregma) was implanted into the lateral ventricle of the right hemisphere. During preparation, the placement of electrodes was adjusted to get maximal populationspike amplitudes (PSA) for a given test pulse intensity $(0.4 \mathrm{~mA}$; A-M Systems, Isolated Pulse Stimulator, Model 2100). The animals were allowed at least 1 wk to recover from surgery.

Connecting the electrodes to a swivel by a flexible cable allowed the rats to move freely in a recording box $(40 \mathrm{~cm}$ $\times 40 \mathrm{~cm} \times 40 \mathrm{~cm})$. The responses were amplified (differential amplifier, Inh, Science Products, Hochheim) transformed by an analog/digital interface (CED 1401+, Cambridge Electronic Design) and stored on a PC. An input/ output curve was performed $16-17 \mathrm{~h}$ before tetanus by recording the average of three stimuli with an intensity of $0.1-0.8$ $\mathrm{mA}$ in steps of $0.1 \mathrm{~mA}$. Biphasic constant current pulses (0.1 msec per half-wave) were applied to the perforant path in order to evoke DG field potentials of about $40 \%$ of the maximum PSA. After recording a stable baseline for $1 \mathrm{~h}$ (every 15 min), early-LTP was induced by weak tetanic bursts (three bursts of 15 pulses of $200 \mathrm{~Hz}$ with $0.1 \mathrm{msec}$ duration of each stimulus and 10-sec interburst interval) at the same stimulus intensity as used for the test stimuli $(0.2-0.4 \mathrm{~mA})$. Initially, after 2 min and then every 15 min after tetanization, five test stimuli (10-sec interpulse intervals) were applied, and the mean values of field potentials were stored for $8 \mathrm{~h}$. For analysis and presentation, 1 -h values were averaged over every four 15 -min values. The next day, four $15-\mathrm{min}$ values were averaged for a 24 -h value. The 2 -min value controlled for the achievement of a sufficient initial potentiation.

\section{Holeboard apparatus and procedure}

The test apparatus consisted of a black board $(1 \mathrm{~m} \times 1 \mathrm{~m})$ with 36 regularly arranged holes $(6 \mathrm{~cm}$ dia, $8 \mathrm{~cm}$ deep) and transparent $27-\mathrm{cm}$-high Plexiglas walls around it. (COGITAT by Cognitron). Distal visual cues consisted of equipment; additional cues were fixed on the outside of the Plexiglas walls. The holes were equipped with photo beams at the surface, in the middle, and on the ground and were baited with standard food pellets (dustless precision pellets, $45 \mathrm{mg}$, BioServ). Signals of the photo beams were registered, counted, and stored on a PC by RatMemory V2.4 software (Heim et al. 2002).

During experiments, animals were transferred into a start box in the holeboard room. The box was opened and the animal entered the test arena. Breakings of the surface beams were registered as "inspections"; breaking of the beams in the middle of the holes as "visits." Removal of the pellets from the ground was registered as "findings." A trial was automatically stopped after 2 min or when the animal had found all pellets. The time to find all pellets (latencies), the working-memory errors (inspecting or visiting a hole that has been baited but that was already inspected or visited and the pellet picked up during a specific trial), and the reference-memory errors (inspecting or visiting a hole that was unbaited) were counted. After each trial the animal was taken back to the recording chambers. The board was cleaned with acetic acid (3\%) after each block of trials and after an individual trial if an animal urinated or defecated during that trial, to eliminate olfactory cues. The holeboard was not cleaned after every trial, in order to elude avoidance reactions and conditioning of the animals to the smell of acetic acid. Beneath the holeboard there was a second board on which food pellets were scattered randomly to avoid odor information from baited holes.

A total of 90 animals with electrode implantations were used. Four groups of animals received a spatial training on a fixed pattern of baited holes (five holes out of 36, Fig. 1A) of five $(n=12)$, seven $(n=9)$, eight $(n=9)$, and 10 trials $(n=9)$, respectively with 15-min intertrial intervals, following the experimental protocol given in Figure 6.

Four groups of animals served as controls: (1) Animals that remained in the recording chamber throughout the experiment (chamber control, $n=12$ ); (2) animals that were exposed to the holeboard the very first time (novelty control, $n=7$ ); (3) animals

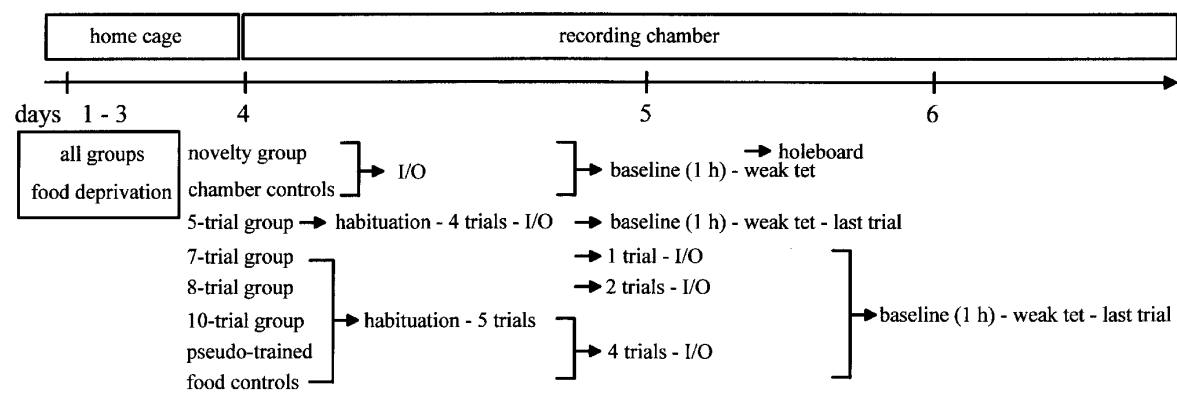

Figure 6. Experimental procedure. The animals were food-deprived over $3 \mathrm{~d}$ to reach $85 \%$ of the initial body weight. On day 4, the animals were transferred to the recording chamber located in a different laboratory room. Fifteen min after weak tetanization (weak tet), the animals received the last holeboard trial and afterwards were transferred back to the recording chamber. LTP was registered for $8 \mathrm{~h}$. On the following day, a 24-h value was recorded. I/O: Input-output curves. The box at the top indicates the time the animals spent in their home cages in the keeping room, and in the recording chamber. 
pre-trained by nine trials with changing patterns of baited holes after each trial (pseudo-trained control, $n=8$ ); and (4) animals transferred 10 times to the board where food pellets were provided without spatial training (food control, $n=6$ ). Tetanization took place $15 \mathrm{~min}$ before the last transfer to the holeboard (between 8:00 a.m. and 9:30 a.m. to consider the diurnal rhythm of corticosterone titers). During training and testing, animal's access to water was ad libitum. Two food pellets/day were provided at random timepoints to avoid anticipation, but with enough time (several hours) between the delivery of food pellets and training to avoid effects on the motivation for food searching in the holeboard.

\section{Hormone analysis}

For the analysis of the hormonal status under the different experimental conditions, we used sets of animals that did not undergo an operation but experienced the same treatment as those rats with electrode implantations. We used holeboard-naive animals (novelty group, $n=8$ ) that were exposed to the holeboard for 2 min and another group $(n=8)$ that experienced a 10-trial training on a fixed pattern of pellets. These rats were decapitated within $10 \mathrm{sec}, 15 \mathrm{~min}$ after behavioral manipulations, and trunk blood was sampled in Eppendorf tubes and allowed to coagulate on ice. The blood was then centrifuged and the serum was stored at $-20^{\circ} \mathrm{C}$. Samples were analyzed by radioimmunoassay. Fooddeprived $(n=8)$ and nondeprived $(n=6)$ animals that remained in the recording box served as control groups. Blood sampling always took place between 10:00 a.m. and 11:00 a.m. to avoid interference with the diurnal rhythm of corticosterone.

\section{Pharmacology}

Anisomycin (Sigma), a reversible translation inhibitor, was applied at a dose that has been shown not to affect early-LTP $(240$ $\mu \mathrm{g}$ i.c.v., $5 \mu \mathrm{L}$ over a 5-min period; Straube et al. 2003b). Two groups of animals $(n=9$ each) that underwent treatment identical to that of the 10-trial experimental group were injected with anisomycin or with saline (i.c.v.) $1 \mathrm{~h}$ before starting the second training session, (i.e., before Trial 6) and received a weak tetanus before Trial 10.

\section{Statistics}

The general linear model for repeated measures was used for group comparisons in LTP, and one-way ANOVA was used for comparisons of behavior and hormones (Dunnett's T-test as post hoc test for pairwise comparisons). Differences in behavior for individual trials between experimental and pseudo-trained animals were tested with Mann-Whitney U-tests ( $P$-values were corrected with the Bonferroni procedure). All tests were two-tailed, and the level of significance was set at $P \leq 0.05$. In all figures the means and standard error of the mean (s.e.m.) are given.

\section{Acknowledgments}

This study was supported by the Land Saxony-Anhalt (LSA3475A/1102M). We thank Dr. Volker Stefanski for analyzing the blood samples.

\section{References}

Abel, T., Nguyen, P.V., Barad, M., Deuel, T.A., Kandel, E.R., and Bourtchouladze, R. 1997. Genetic demonstration of a role for PKA in the late phase of LTP and in hippocampus-based long-term memory. Cell 88: 615-626.

Almaguer-Melian, W., Martinez-Marti, L., Frey, J.U., and Bergado, J.A. 2003. The amygdala is part of the behavioural reinforcement system modulating long-term potentiation in rat hippocampus. Neuroscience 119: $319-322$.

Bach, M.E., Barad, M., Son, H., Zhuo, M., Lu, Y.F., Shih, R., Mansuy, I., Hawkins, R.D., and Kandel, E.R. 1999. Age-related defects in spatial memory are correlated with defects in the late phase of hippocampal long-term potentiation in vitro and are attenuated by drugs that enhance the cAMP signalling pathway. Proc. Natl. Acad. Sci. 96: 5280-5285.

Bailey, C.H., Giustetto, M., Huang, Y.Y., Hawkins, R.D., and Kandel, E.
2000. Is heterosynaptic modulation essential for stabilizing hebbian plasticity and memory? Nat. Rev. 1: $11-20$.

Barros, D.M., Mello e Souza, T., De David, T., Choi, H., Aguzzoli, A., Madche, C., Ardenghi, P., Medina, J.H., and Izquierdo, I. 2001. Simultaneous modulation of retrieval by dopaminergic $\mathrm{D}(1)$, $\beta$-noradrenergic, serotonergic-1A and cholinergic muscarinic receptors in cortical structures of the rat. Behav. Brain. Res. 124: 1-7.

Bilang-Bleuel, A., Rech, J., De Carli, S., Holsboer, F., and Reul, J.M. 2002. Forced swimming evokes a biphasic response in CREB phosphorylation in extrahypothalamic limbic and neocortical brain structures in the rat. Eur. J. Neurosci. 15: 1048-1060.

Braunewell, K.H. and Manahan-Vaughan, D. 2001. Long-term depression: A cellular basis for learning? Rev. Neurosci. 12: 121-140.

Castellano, C., Cabib, S., Puglisi-Allegra, S., Gasbarri, A., Sulli, A., Pacitti, C., Introini-Collison, I.B., and McGaugh, J.L. 1999. Strain-dependent involvement of D1 and D2 dopamine receptors in muscarinic cholinergic influences on memory storage. Behav. Brain. Res. 98: $17-26$.

Debiec, J., LeDoux, J.E., and Nader, K. 2002. Cellular and systems reconsolidation in the hippocampus. Neuron 36: 527-538.

Doyère, V., Redini-Del Negro, C., Dutrieux, G., Le Floch, G., Davis, S., and Laroche, S. 1995. Potentiation or depression of synaptic efficacy in the dentate gyrus is determined by the relationship between the conditioned and unconditioned stimulus in a classical conditioning paradigm in rats. Behav. Brain. Res. 70: 15-29.

Doyère, V., Srebro, B., and Laroche, S. 1997. Heterosynaptic LTD and depotentiation in the medial perforant path of the dentate gyrus in the freely moving rat. J. Neurophysiol. 77: 571-578.

Duffy, S.N., Craddock, K.J., Abel, T., and Nguyen, P.V. 2001. Environmental enrichment modifies the PKA-dependence if hippocampal LTP and improves hippocampus-dependent memory. Learn. Mem. 8: 26-34.

Flexner, L.B., Flexner, J.B., De La Haba, G., and Roberts, R.B. 1965. Loss of memory as related to inhibition of cerebral protein synthesis. J. Neurochem. 12: 535-541.

Frey, U. and Morris, R.G. 1997. Synaptic tagging and long-term potentiation. Nature 385: $533-536$

Frey, U., Krug, M., Reymann, K.G., and Matthies, H. 1988. Anisomycin, an inhibitor of protein synthesis, blocks late phases of LTP phenomena in the hippocampal CA1 region in vitro. Brain Res. 452: $57-65$.

Frey, S., Bergado-Rosado, J., Seidenbecher, T., Pape, H.C., and Frey, J.U. 2001. Reinforcement of early long-term potentiation (early-LTP) in dentate gyrus by stimulation of the basolateral amygdala: Heterosynaptic induction mechanisms of late-LTP. J. Neurosci. 21: $3697-3703$.

Frey, S., Bergado, J.A., and Frey, J.U. 2003. Modulation of late phases of long-term potentiation in rat dentate gyrus by stimulation of the medial septum. Neurosci. 118: 1055-1062.

Gooney, M., Shaw, K., Kelly, A., O'Mara, S.M., and Lynch, M.A. 2002. Long-term potentiation and spatial learning are associated with increased phosphorylation of TrkB and extracellular signal-regulated kinase (ERK) in the dentate gyrus: Evidence for a role for brain-derived neurotrophic factor. Behav. Neurosci. 116: 455-463.

Grecksch, G. and Matthies, H. 1980. Two sensitive periods for the amnesic effect of anisomycin. Pharmacol. Biochem. Behav. 12: $663-665$.

Heiderstadt, K.M., McLaughlin, R.M., Wright, D.C., Walker, S.E., and Gomez-Sanchez, C.E. 2000. The effect of chronic food and water restriction on open-field behaviour and serum corticosterone levels in rats. Lab. Anim. 34: 20-28.

Heim, C., Pardowitz, I., Sieklucka, M., Kolasiewicz, W., Sontag, T., and Sontag, K.H. 2002. The analysis system COGITAT for the study of cognitive deficiencies in rodents. Behav. Res. Methods Instrum. Comput. 32: 140-156.

Huang, Y.Y., Li, X.C., and Kandel, E.R. 1994. cAMP contributes to mossy fiber LTP by initiating both a covalently mediated early phase and macromolecular synthesis-dependent late phase. Cell 79: 69-79.

Hugin-Flores, M.E., Steimer, T., Aubert, M.L., and Schulz, P. 2004. Mineralo- and glucocorticoid receptor mrnas are differently regulated by corticosterone in the rat hippocampus and anterior pituitary. Neuroendocrinol. 79: 174-184.

Impey, S., Mark, M., Villacres, E.C., Poser, S., Chavkin, C., and Storm, D.R. 1996. Induction of CRE-mediated gene expression by stimuli that generate long-lasting LTP in area CA1 of the hippocampus. Neuron 16: 973-982.

Izquierdo, L.A., Barros, D.M., Vianna, M.R., Coitinho, A., deDavid, E., Silva, T., Choi, H., Moletta, B., Medina, J.H., and Izquierdo, I. 2002. Molecular pharmacological dissection of short- and long-term memory. Cell. Mol. Neurobiol. 22: 269-287.

Jaffard, R., Vouimba, R.M., Marighetto, A., and Garcia, R. 1996 Long-term potentiation and long-term depression in the lateral 
septum in spatial working and reference memory. J. Physiol. Paris 90: $339-341$

Jeffery, K.J. 1997. LTP and spatial learning-Where to next? Hippocampus 7: 95-110.

Jones, M.W., Errington, M.L., French, P.J., Fine, A., Bliss, T.V.P., Garel, S., Charnay, P., Bozon, B., Laroche, S., and Davis, S. 2001. A requirement for the immediate early gene Zif268 in the expression of late-LTP and long-term memories. Nat. Neurosci. 4: 289-296.

Jurcovicova, J., Stancikova, M., Svik, K., Ondrejickova, Krsova, D., Seres, J., and Rokyta, R. 2001. Stress of chronic food restriction attenuates the development of adjuvant arthritis in male Long Evans rats. Clin. Exp. Rheumatol. 19: 371-376.

Karandrea, D., Kittas, C., and Kitraki, E. 2002. Forced swimming differentially affects male and female brain corticosteroid receptors. Neuroendocrinol. 75: 217-226.

Kelleher III, R.J., Govindarajan, A., and Tonegawa, S. 2004. Translational regulatory mechanisms in persistent forms of synaptic plasticity. Neuron 44: 59-73.

Kemp, A. and Manahan-Vaughan, D. 2004. Hippocampal long-term depression and long-term potentiation encode different aspects of novelty acquisition. Proc. Natl. Acad. Sci. 101: 8192-8197.

Kentros, C.G., Agnihotri, N.T., Streater, S., Hawkins, R.D., and Kandel, E.R. 2004. Increased attention to spatial context increases both place field stability and spatial memory. Neuron 42: 283-295.

Kikusui, T., Aoyagi, A., and Kaneko, T. 2000. Spatial working memory is independent of hippocampal CA1 long-term potentiation in rats. Behav. Neurosci. 114: 700-706.

Kitchigina, V., Vankov, A., Harley, C., and Sara, S.J. 1997. Novelty-elicited, noradrenaline-dependent enhancement of excitability in the dentate gyrus. Eur. J. Neurosci. 9: 41-47.

Kiss, A., Jezova, D., and Aguilera, G. 1994. Activity of the hypothalamic pituitary adrenal axis and sympathoadrenal system during food and water deprivation in the rat. Brain Res. 663: 84-92.

Korz, V. and Frey, J.U. 2003a. Stress-related modulation of hippocampal long-term potentiation in rats: Involvement of adrenal steroid receptors. J. Neurosci. 23: 7281-7287.

. 2003b. Stress and novelty related modulation of rat hippocampal LTP. Abstract Viewer Itinerary Planner, Program No. 623.17. Society for Neuroscience, Washington, D.C.

Krug, M., Lossner, B., and Ott, T. 1984. Anisomycin blocks the late phase of long-term potentiation in the dentate gyrus of freely moving rats. Brain Res. Bull. 13: 39-42.

Kulla, A. and Manahan-Vaughan, D. 2002. Modulation by serotonin 5-HT(4) receptors of long-term potentiation and depotentiation in the dentate gyrus of freely moving rats. Cereb. Cortex 12: 150-162.

Litvin, O.O. and Anokhin, K.V. 1999. The mechanisms of memory reorganization during the retrieval of acquired behavioral experience in chicks: The effects of protein synthesis blockade in the brain. $Z h$. Vyssh. Nerv. Deiat Im. I. P. Pavlova 49: 554-565.

Manahan-Vaughan, D. and Kulla, A. 2003. Regulation of depotentiation and long-term potentiation in the dentate gyrus of freely moving rats by dopamine D2-like receptors. Cereb. Cortex 13: 123-135.

Matthies, H., Ruethrich, H., Ott, T., Matthies, H.K., and Matthies, R. 1986. Low frequency perforant path stimulation as a conditioned stimulus demonstrates correlations between long-term synaptic potentiation and learning. Physiol. Behav. 36: 811-821.

Meyer, U., van Kampen, M., Isovich, E., Flugge, G., and Fuchs, E. 2001. Chronic psychosocial stress regulates the expression of both GR and MR mRNA in the hippocampal formation of tree shrews. Hippocampus 11: 329-336.

Morris, R.G.M. and Frey, U. 1997. Hippocampal synaptic plasticity: Role in spatial learning or the automatic recording of attended experience? Phil. Trans. R. Soc. Lond. B 352: 1489-1503.

Moser, E.I., Moser, M.B., and Andersen, P. 1994. Potentiation of dentate synapses initiated by exploratory learning in rats: Dissociation from brain temperature, motor activity, and arousal. Learn. Mem. 1: $55-73$.

Murchison, C.F., Zhang, X.-Y., Zhang, W.-P., Ouyang, M., Lee, A., and Thomas, S.A. 2004. A distinct role for norepinephrine in memory retrieval. Cell 117: 131-143.
Myhrer, T. 2003. Neurotransmitter systems involved in learning and memory in the rat: A meta-analysis based on studies of four behavioral tasks. Brain. Res. Rev. 41: 268-287.

Nader, K., Schafe, G.E., and LeDoux, J.E. 2000. Fear memories require protein synthesis in the amygdala for reconsolidation after retrieval. Nature 406: $722-726$.

Nakao, K., Ikegaya, Y., Yamada, M.K., Nishiyama, N., and Matsuki, N. 2002. Hippocampal long-term depression as an index of spatial working memory. Eur. J. Neurosci. 16: 970-974.

Otani, S. and Abraham, W.C. 1989. Inhibition of protein synthesis in the dentate gyrus, but not the entorhinal cortex, blocks maintenance of long-term potentiation in rats. Neurosci. Lett. 106: $175-180$.

Otani, S. and Ben-Ari, Y. 1993. Biochemical correlates of long-term potentiation in hippocampal synapses. Int. Rev. Neurobiol. 35: 1-41.

Phillips, A.G., Ahn, S., and Floresco, S.B. 2004. Magnitude of dopamine release in medial prefrontal cortex predicts accuracy of memory on a delayed response task. J. Neurosci. 24: 547-553.

Przybyslawski, J., Roullet, P., and Sara, S.J. 1999. Attenuation of emotional and nonemotional memories after their reactivation: Role of $\beta$ adrenergic receptors. J. Neurosci. 19: 6623-6628.

Richter-Levin, G. and Akirav, I. 2003. Emotional tagging of memory formation-In the search for neural mechanisms. Brain Res. Brain Res. Rev. 43: 247-256.

Richter-Levin, G., Errington, M.L., Maegawa, H., and Bliss, T.V. 1994. Activation of metabotropic glutamate receptors is necessary for long-term potentiation in the dentate gyrus and for spatial learning. Neuropharmacol. 33: 853-857.

Rosenzweig, E.S., Barnes, C.A., and McNaughton, B.L. 2002. Making rooms for new memories. Nat. Neurosci. 5: 6-8.

Sara, S.J. 2000. Retrieval and reconsolidation: Toward a neurobiology of remembering. Learn. Mem. 7: 73-84.

Saucier, D. and Cain, D.P. 1995. Spatial learning without NMDA receptor-dependent long-term potentiation. Nature 378: 186-189.

Schulz, S., Siemer, H., Krug, M., and Höllt, V. 1999. Direct evidence for biphasic cAMP responsive element-binding protein phosphorylation during long-term potentiation in the rat dentate gyrus in vivo. $J$. Neurosci. 19: 5683-5692.

Schulz, D., Huston, J.P., Jezek, K., Haas, H.L., Roth-Harer, A., Selbach O., and Luhmann, H.J. 2002. Water maze performance, exploratory activity, inhibitory avoidance and hippocampal plasticity in aged superior and inferior learners. Eur. J. Neurosci. 16: 2175-2185.

Sebaai, N., Lesage, J., Vieau, D., Alaoui, A., Dupouy, J.P., and Deloof, S. 2001. Altered control of the hypothalamo-pituitary-adrenal axis in adult male rats exposed perinatally to food deprivation and/or dehydration. Neuroendocrinol. 76: 243-253.

Seidenbecher, T., Reymann, K.G., and Balschun, D. 1997. A post-tetanic time window for the reinforcement of long-term potentiation by appetitive and aversive stimuli. Proc. Natl. Acad. Sci. 94: 1494-1499.

Shors, T. 2004. Learning during stressful times. Learn. Mem. 11: $137-144$.

Straube, T., Korz, V., and Frey, J.U. 2003a. Bidirectional modulation of long-term potentiation by novelty-exploration in rat dentate gyrus. Neurosci. Lett. 344: 5-8.

Straube, T., Korz, V., Balschun, D., and Frey, J.U. 2003b. Requirement of $\beta$-adrenergic receptor activation and protein synthesis for LTP-reinforcement by novelty in rat dentate gyrus. J. Physiol. 552: $953-960$

Tronel, S., Feenstra, M.G., and Sara, S.J. 2004. Noradrenergic action in prefrontal cortex in the late stage of memory consolidation. Learn. Mem. 11: $453-458$.

Villarreal, D.M., Do, V., Haddad, E., and Derrick, B.E. 2001. NMDA receptor antagonists sustain LTP and spatial memory: Active processes mediate LTP decay. Nat. Neurosci. 5: 48-52.

Woo, N.H. and Nguyen, P.V. 2003. Protein synthesis is required for synaptic immunity to depotentiation. J. Neurosci. 23: 1125-1132.

Received September 14, 2004; accepted in revised form December 16, 2004. 


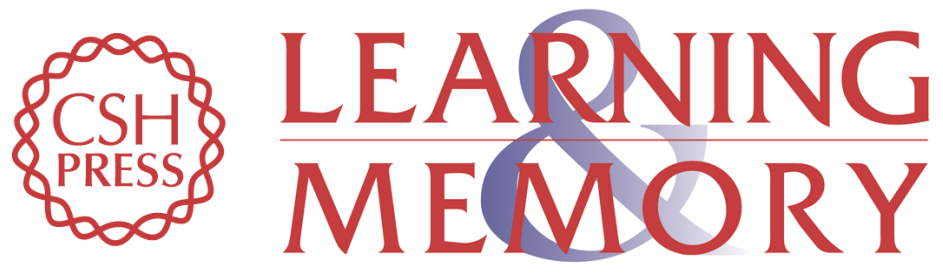

\section{Reinforcement of rat hippocampal LTP by holeboard training}

Shukhrat Uzakov, Julietta U. Frey and Volker Korz

Learn. Mem. 2005, 12:

Access the most recent version at doi:10.1101/lm.89305

References This article cites 66 articles, 14 of which can be accessed free at: http://learnmem.cshlp.org/content/12/2/165.full.html\#ref-list-1

License

Email Alerting Receive free email alerts when new articles cite this article - sign up in the box at the Service top right corner of the article or click here. 